\title{
Biventricular with multi-site left-ventricular pacing for the management of refractory ventricular tachycardia in a patient with narrow complex qrs -
} case report

\begin{abstract}
A patient with non-ischemic cardiomyopathy, advanced heart failure, narrow complex QRS, $>40 \%$ premature ventricular complexes (PVCs) and recurrent sustained monomorphic ventricular tachycardia (VT) episodes had failed pharmacotherapy and recurrent VT ablations. A trans-venous biventricular defibrillator with multi-site LV pacing (MS-CRTD) was implanted (i.e. two left ventricular (LV) leads through the coronary sinus and one right ventricular lead). Immediate reduction in PVCs burden was inspected. Within one year PVCS and VTs subsided, antiarrhythmic medications were discontinued, and LV function and heart failure symptoms improved dramatically. The case suggests that biventricular multi-site LV pacing may serve as an alternative option for treating refractory VT. Further studies to assess this technique and the underlying mechanism are warranted.
\end{abstract}

Keywords: multi-site pacing, ventricular tachycardia, heart failure, biventricular pacing, left ventricular pacing
Volume 8 Issue 2 - 2017

\author{
Avishag Laish Farkash,Vladimir Khalameizer, \\ Amos Katz \\ Department of Cardiology, Barzilai University Medical Center, \\ Ashkelon, and Faculty of Health Sciences, Ben-Gurion University \\ of the Negev, Israel
}

Correspondence: Avishag Laish-Farkash, Department of Cardiology, Barzilai Medical Center, 2 Hahistadrut Street, Ashkelon 78278, Israel, Tel +972-53-7678726, Fax +972-86745431,Email avishagl@inter.net.il

Received: January 25, 2017 | Published: February 07, 2017

\section{Case presentation}

A 59-year-old male was admitted to our hospital with heart failure (HF) exacerbation and sustained monomorphic ventricular tachycardia (SMVT) episodes. The patient was known to have had severe non-ischemic left ventricular (LV) dysfunction for the past four years. His history was also remarkable for diabetes mellitus, hypertension, and gastric banding. The patient had documentation of LV ejection fraction (EF) of $20 \%$, diffuse hypokinesis and LV end systolic and diastolic volumes (LVESV/LVEDV): 134/172ml. His coronary catheterization demonstrated non-significant coronary artery disease and he had a normal cardiac biopsy. Over those years the patient was hospitalized frequently due to worsening HF symptoms and was graded as New York Heart Association (NYHA) functional class III. His baseline ECG showed normal sinus rhythm with QRS duration of $92 \mathrm{~ms}$. Recurrent $24 \mathrm{~h}$-Holter monitoring showed $>40 \%$ $(>50,000)$ PVCs with a morphology of intermediate right bundle (RS in V1) and right-inferior axis, with multiple episodes of SMVT. Tachycardia-induced cardiomyopathy was suspected.

Sixteen months prior to current hospital admission the patient had underwent an electrophysiology study (in another medical center) in order to map and ablate the arrhythmia; endocardial, aortic cusps and coronary sinus (CS) mapping of PVCs using a 4F CARDIMA catheter for the CS and CARTO 3-D mapping system were employed. Earliest activation was located to the middle part of the great cardiac vein (anterior interventricular branching) with 12/12 pace mapping, $50 \mathrm{~ms}$ prior to QRS. The operator was unable to pass an ablation catheter forward to distal CS and the procedure was discontinued. A few months later the patient underwent another ablation attempt: an epicardial catheter was located opposite to the earliest point of the CARDIMA CS catheter. RF ablation at that area abolished the PVCs, but PVCs resumed after a few hours. Several months later the patient was referred for a third procedure. Conscious sedation caused temporary disappearance of PVCs and no inducibility of VT. Pace mapping was not perfect and the procedure was discontinued. The patient refused further attempts.
He was treated with maximal tolerated doses of sotalol, quinidine and then was fully loaded with amiodarone (each antiarrhytmic drug at a time) along with beta blockers. Despite those antiarrhythmic drugs (several months each), the patient had recurrent hospitalizations for HF exacerbations and SMVT episodes. The patient had an indication for ICD implantation for secondary prevention and although the patient had narrow QRS, we decided to implant a CRT-D because we anticipated a need for overdrive RV pacing in a patient with an upfront severe LV dysfunction and an expected further LV function deterioration. Furthermore, the final decision was to implant two LV leads at different locations, aiming for a stronger possible antiarrhythmic effect-as proposed in the discussion section. The patient gave his written informed consent to the procedure. A transvenous biventricular ICD (PROTECTA, Model D364TRG, Medtronic Inc., Minneapolis, MN, USA) was implanted and was connected to one right atrial (RA) lead, one right ventricular (RV) lead and two left ventricular (LV) leads. RV lead (bipolar, active fixation, Model $6947-65 \mathrm{~cm}$, Medtronic Inc.) was positioned through a left cephalic approach in the RV apex; the other leads were inserted through left subclavian approach. RA lead (bipolar, passive fixation, Model 5592$53 \mathrm{~cm}$, Medtronic Inc.) was positioned in the appendage. One LV lead (bipolar, Model 4194-88cm, Medtronic Inc.) was positioned in an anterior branch of the coronary sinus system (Figure 1, LV1). The second LV lead (bipolar lead, Model 1258T, St. Jude Medical Inc., St. Paul, MN, USA) was positioned in a posterior branch of the coronary sinus system (Figure 1, LV2). The two LV leads were connected through a bipolar Y-connector (thus, every biventricular pacing (BVP) was a multisite BVP). The device was programmed with a short atrioventricular interval to assure BVP. LV pacing was simultaneous in the two LV leads (through a Y-connector). LV-RV VV interval could be programmed but was set to LV preceding RV in $10 \mathrm{msec}$ (the interval that showed the thinnest QRS complex). Immediately post-implantation of LV leads and before connecting the leads to the device the PVCs still existed - ruling out the possibility of traumatic injury of the VT focus during implantation as a possible mechanism of VT abolishment. After connecting the leads to the device it was 
programmed to multisite biventricular pacing with a lower rate of 80 . 24-hour Holter monitoring one day post-implantation showed $0.1 \%$ VPBs (116/24hours) with no VT episodes (Figure 2). In order to evaluate the role of overdrive pacing or long-short cycle prevention in the antiarrhythmic effect, a day later the device was programmed AAI with a lower rate of $85 \mathrm{bpm}$. A consecutive 24-h Holter monitoring showed 7.3\% PVCs (9141/24hours) and no VT episodes. The device was then programmed BVP at a lower rate of DDD-50 (to prevent overdrive pacing). Further 24h-Holter monitoring showed $1 \%$ of PVCs (1169/24hours), with no VT episodes (Figure 2). Periodic follow-up in the ICD clinic showed no more VT episodes since implantation. Antiarrhythmic drugs were discontinued later on, and the patient was never admitted for hospitalization. There were no changes in his medications (ACE-I and Carvedilol 25mg BID), though his diuretics were reduced over time. HF symptoms were improved dramatically, with NYHA functional class II. Echocardiography one year post-implantation showed major improvement: LVEF 33\% and LVESV/LVEDV of $71 / 107 \mathrm{ml}$. The BVP was turned off after a year of arrhythmic, clinical and echocardiographic improvement. A consecutive 24-h Holter monitoring showed 8\% PVCs (9915/24 hours) and 9 VT episodes (Figure 2). The patient felt weakness, dizziness and shortness of breath. All complaints had resolved after turning the BVP on again. A follow-up echocardiography one year later showed $\mathrm{EF}$ of $45 \%$.
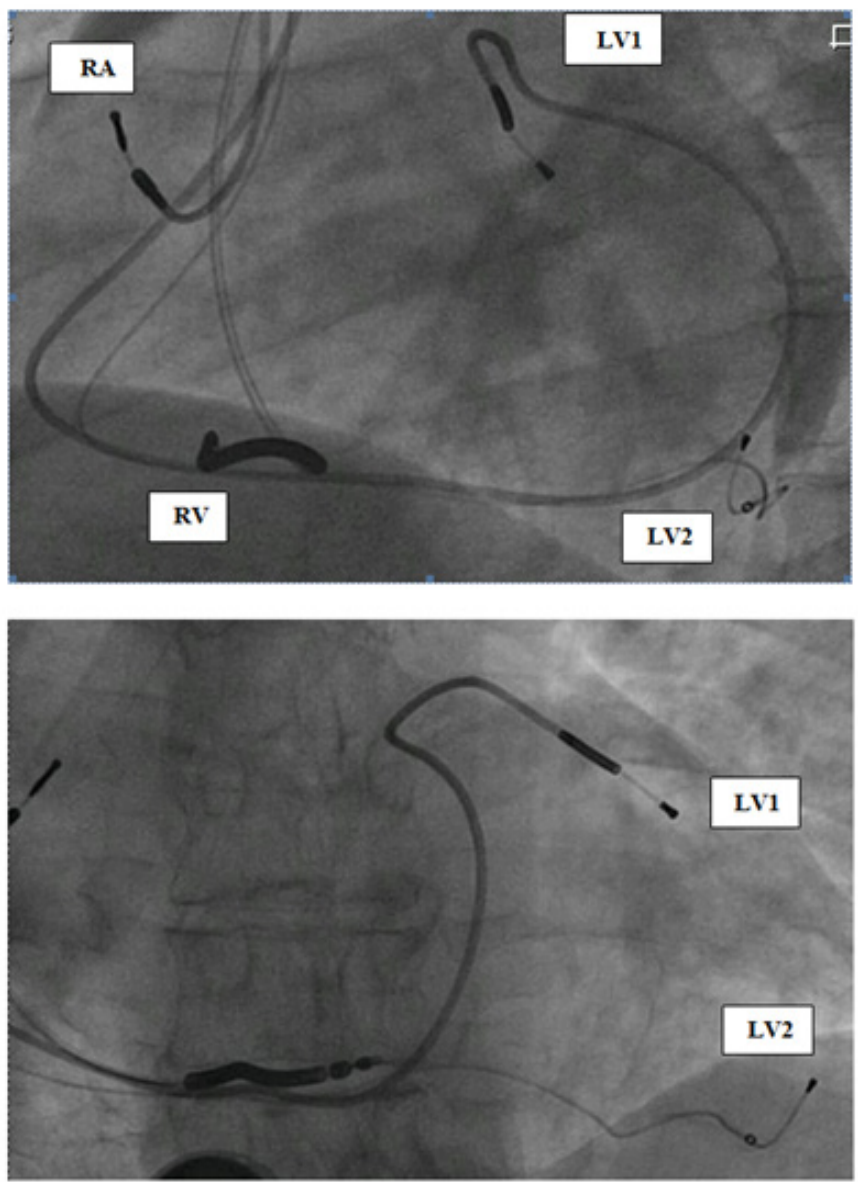

Figure I Fluoroscopy LAO and AP views showing the four leads. LVI: Left Ventricular Lead Positioned in Anterior Branch of Coronary Sinus; LV2: Left Ventricular Lead Positioned in Posterior Vein; RA: Right Atrial Leads; RV: Right Ventricular Lead.

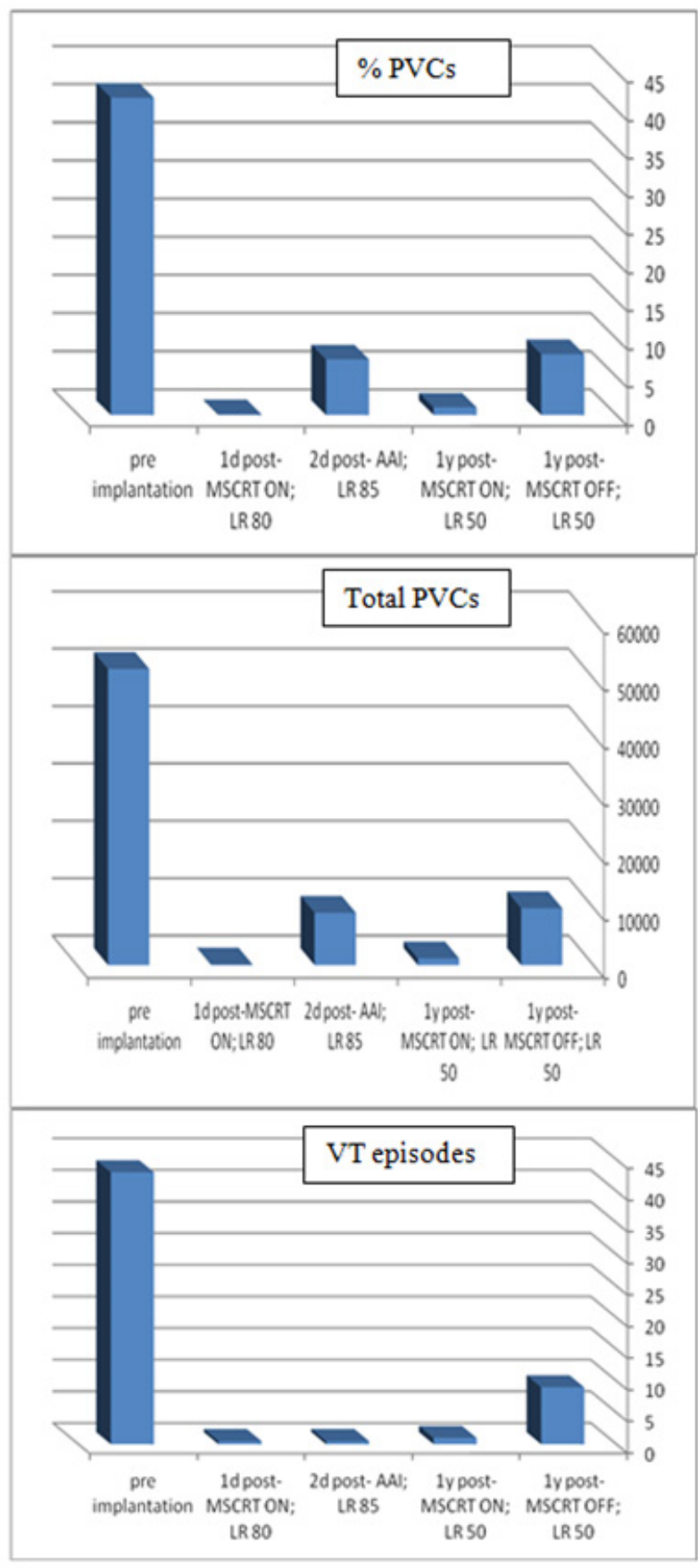

Figure 224h-Holter monitoring records of the patient (see full text for details) Id post-MSCRT: Immediately post-implantation the device was programmed BVP (with multi-site LV pacing via Y-connector) with lower rate of DDD-80.

\section{Discussion}

While CRT could increase the dispersion of repolarization and induce malignant ventricular tachyarrhythmias in a small subgroup of patients, ${ }^{1,2}$ over the past few years, different studies have shown that apart from hemodynamic benefits, BVP may reduce ventricular tachyarrhythmia susceptibility in HF patients with conventional indications for CRT. ${ }^{3-6}$ This suppressive effect was also shown in electrophysiologic studies ${ }^{7}$. As opposed to HF symptoms, the incidence of ventricular arrhythmia is not different based on the LV 
lead position in patients with CRT. ${ }^{8}$ The MADIT-CRT study showed that the reduction in the risk of ventricular tachyarrhythmias that was observed in the study population was shown in LBBB but not RBBB or IVCD patients. ${ }^{6}$ Patients with narrow-complex were not included in this study. Acosta et al., ${ }^{9}$ described a case in which a patient with non-ischemic cardiomyopathy and LBBB developed an electrical storm despite having a biventricular ICD implanted and being on antiarrhythmic therapy. ${ }^{9}$ Adding a new LV lead to provide multi-site LV pacing on top of BVP resulted in suppression of the ventricular arrhythmia, narrowing of the QRS complex, and improvement of HF functional class. Our case report describes a patient with nonischemic-, and probably some component of tachycardia inducedcardiomyopathy, with narrow complex QRS, who failed VT ablation and antiarrhythmic drugs. Dual-site LV pacing on top of BVP caused immediate suppression of VT episodes and PVCs with subsequent further improvement of LVEF and functional class.

The underlying mechanism could be additive to the antiarrhythmic mechanism of BVP since the patient had narrow QRS and an immediate suppression of the VT post-implantation as well (traumatic injury to VT focus and overdrive pacing as the underlying mechanisms were ruled out). The overdrive issue, which had some role (reduction but not abolishment of PVCs) during AAI pacing at LR of $85 \mathrm{bpm}$, was mostly ruled out by programming the lower rate at DDD50 with BVP (i.e. actually working as VDD), yet the arrhythmia was still suppressed. The reduction (but not suppression) in the amount of PVCs after one year of multi-site pacing (when BVP was turned off) could be attributed to the major improvement in LVEF.

A change in excitation wave due to pacing could be a possible reason for the disappearance of PVCs and VT episodes. A direct electrophysiological effect due to a more homogeneous depolarization as well as reduction of the dispersion of repolarization cannot be ruled out as an underlying mechanism. ${ }^{10}$ Restivo et al., ${ }^{11}$ demonstrated a direct anti-arrhythmic effect of dual site ventricular stimulation in a canine post-infarction model. Properly sequenced stimulation at sites of functional refractoriness prevented the induction of reentry in that model. If this is the mechanism, adding a second LV lead in those rare cases of pro-arrhythmic BVP could be a possible solution, since these patients have other indications for CRT and could benefit from the device for HF and survival issues.

Multi-site LV pacing could also improve hemodynamics by providing better synchrony of contraction, reduction of shearing forces, stretch ,and sympathetic activity, thus providing an anti-arrhythmic "vicinity" ${ }^{12}$ Pappone et al., ${ }^{13}$ demonstrated in an acute hemodynamic study of patients with classic indications for CRTD, a positive immediate effect (increase in LV dP/dt max) of dual-site LV pacing, compared with single-site LV pacing. ${ }^{13}$ The author concluded that improved ventricular synchrony, evaluated by paced QRS narrowing, may account for the additional benefit of dual - over single-site - LV pacing. In conclusion, as far as we know this is the first report of a patient with non-ischemic cardiomyopathy and narrow complex QRS in whom multi-site LV pacing with BVP via a transvenous approach causes an immediate reduction of his refractory PVCs and VT episodes and in whom improvement of LVEF and NYHA functional class resulted. Transvenous dual-site LV pacing could be a treatment option for those patients with refractory ventricular arrhythmias who failed antiarrhythmic and ablation therapy. Further studies to assess this technique and the underlying mechanism are warranted.

\section{Acknowledgments}

We thank Michael Glikson MD, the director of Davidai Arrhythmia Center, Leviev Heart Center, Sheba Medical Center, Tel Hashomer, Israel, for supplying the EPS and ablation data of this patient.

\section{Conflicts of interest}

Author declares there is no conflicts opf interest.

\section{Funding}

None.

\section{References}

1. Fish JM, Di Diego JM, Nesterenko V, et al. Epicardial activation of left ventricular wall prolongs QT interval and transmural dispersion of repolarization. Implications for biventricular pacing. Circulation. 2004;109(17):2136-2142.

2. Nayak HM, Verdino RJ, Russo AM, et al. Ventricular tachycardia storm after initiation of biventricular pacing: incidence, clinical characteristics, management, and outcome. J Cardiovasc Electr. 2008;19(7):708-715.

3. Walker S, Levy T, Rex S, et al. Usefulness of suppression of ventricular arrhythmia by biventricular pacing in severe congestive failure. $\mathrm{Am} J$ Cardiol. 2000;86(2):231-233.

4. Ermis C, Seutter R, Zhu AX, et al. Impact of upgrade to cardiac resynchronization therapy on ventricular arrhythmia frequency in patients with implantable cardioverter-defibrillators. $\mathrm{J} \mathrm{Am} \mathrm{Coll} \mathrm{Cardiol.}$ 2005;46(12):2258-2263.

5. Blaschke F, Knaus T, Celebi O, et al. Ventricular tachycardia or ventricular fibrillation occurs less often in patients with left bundle branch block and combined resynchronization and defibrillators than in patients with narrow QRS and conventional defibrillators. Europace. 2012;14(2):224-229.

6. Zareba W, Klein H, Cygankiewicz I, et al. Effectiveness of cardiac resynchronization therapy by QRS morphology in the multicenter automatic defibrillator implantation trial - cardiac resynchronization therapy (MADIT-CRT). Circulation. 2011;123:1061-1072.

7. Zagrodzky JD, Ramaswamy K, Page RL, et al. Biventricular pacing decreases the inducibility of ventricular tachycardia in patients with ischemic cardiomyopathy. Am J Cardiol. 2001;87(10):1208-1210.

8. Kleemann T, Becker T, Strauss M, et al. Impact of left ventricular lead position on the incidence of ventricular arrhythmia and clinical outcome in patients with cardiac resynchronization therapy. J Interv Card Electrophysiol . 2010;28(2):109-116.

9. Acosta H, Pothula VR, Arter J, et al. Transvenous dual site left ventricular pacing plus biventricular pacing for the management of refractory ventricular tachycardia. J Interv Card Electrophysiol. 2006;17(1):73-75.

10. Ploux S, Whinnett $Z$, Bordachar P. Left ventricular endocardial pacing and multisite pacing to improve CRT response. $J$ of Cardiovasc Trans Res. 2012;5(2):213-218.

11. Restivo M, Gough WB, el-Sherif N. Reentrant ventricular rhythms in the late myocardial infarction period: prevention of reentry by dual stimulation during basic rhythm. Circulation . 1988;77(2):429-444.

12. Ploux S, Barandon L, Ritter P, et al. Positive hemodynamic and clinical response to tri-left ventricular pacing in a nonresponder to traditional cardiac resynchronization therapy. Heart Rhythm. 2011;8(2):315-317.

13. Pappone $\mathrm{C}$, Rosanio $\mathrm{S}$, Oreto $\mathrm{G}$, et al. Cardiac pacing in heart failure patients with left bundle branch block: impact of pacing site for optimizing left ventricular resynchronization. Ital Heart J. 2000;1(7): 464-469. 